Jane J. Robinson, Chair

Artiflcial Intelligence Center

SRI International

Nowhere is the tension between the two areas of our field-computation and lingulstics-more apparent than in the issues that arise in connection with parsing natural language input. This panel addresses those issues from both computational and linguistic perspectives. Each panelist has subnitted a position paper on some of the questions that appear below. The questions are loosely grouped in three sections. The E1rst concentrates on the compucational aspect, the second on the linguistic aspect, and the third on the 15 interactions.

A preliminary definition:

For purposes of providing common ground or possibly a common point of departure at the outser, I will define parsing as the assigning of labelled syntactic structure to an input by applying a grammar that defines syntactically well-formed sentences and phrases. Note that the question of whether the grammar does other things as well is left open. In this sense, parsing is distinguished from interpretation, which may take many forms, such as assigning represencacions in an unambiguous formal language and incegrating those representations tnto a data base or inco a hearer's belief system.

The questions:

1. The Computational Perspective:

What useful purposes, if any, are served by distinguishing parsing from interpretation? Is computational effictency Increased? Is system bullding made easier? or is an inststence on parsing a hindrance? (Can we compute an interpretation better without assigning labelled syncactic structures?)

Computational linguists, using avallable computational equipment that is almose exclusively serial in design, have devised parsing algorithms that involve sertal search. Yet it is obvious that many parts of the parsing process could be done in parallel. How mighe notions of parallel processing, VLSI, and the like change our views on parsing?
What mighe motivace our trying to make parsing procedures simulate human behavior, e.g., by incermixing syntactic with semantic and pragmatic processing? And for that matcer, how do we know what human processing is like? Do our intuitions agree and are they to be trusted?

\section{The Linguistic Perspective:}

Have our tools (compucers and formal grammars) warped our views of what human languages and human language processing may be like? What legitimate inferences about human linguistic competence and performance can we draw from our experlences with mechanical parsing of Eormal grammars?

Our most efficient persing algorithms are for context free (and even regular) grammars. Does this suggest that the core of grammars for natural languages $1 \mathrm{~s}$ context free or even regular?

\section{The Interactions:}

Why do we usually have one grammar and procedure for sentence recognition and another grammar and procedure for sentence generation? Do we need a different pair for each direction?

What is the nature of the relationship between a grammar and a procedure for applying it? Are we influenced in the way we devise computational grammars by the algorithms we expect to apply to them? Can a grammar be psychologically valid (validated) 1ndependently of the parsing algorithm that works with it? Can a parsing algorithm be psychologically valid (validated) independently of the grammar?

The discussion to follow:

The position papers will serve to focus the discussion. That discussion may take the form of a debale about the best methods for language processing, but it can also be viewed as a gathering of diverse experiences with Drocessing natural language. 
